\title{
Long-Term Outcomes of Ventral Hernia Repair: An 11-Year Follow-Up
}

Nikita Kadakia ${ }^{1}$, Ross Mudgway ${ }^{2}$, Jonathan Vo ${ }^{3}$, Vinson Vong ${ }^{4}$, Tiffany Seto ${ }^{5}$, Pascal Bortz ${ }^{3}$, Aron Depew 6

1. Surgery, University of California Riverside School of Medicine, Riverside, USA 2. General Surgery, Loma Linda University School of Medicine, Loma Linda, USA 3. General Surgery, Riverside University Health System Medical Center, Riverside, USA 4. Emergency Medicine, University of California Riverside School of Medicine, Riverside, USA 5. Oncology, Kaiser Permanente, San Francisco, USA 6. Surgery, Beaver Medical Group, Redlands, USA

Corresponding author: Nikita Kadakia, nikitakadakia.nk@gmail.com

\begin{abstract}
Background: Ventral hernia repair (VHR) is one of the most common general surgery procedures; however, few studies with long-term follow-up of VHR outcomes exist.

Methods: We performed a retrospective review of VHRs performed from 2000 to 2009 at a single institution. Our primary outcome was recurrence, and secondary outcomes were reoperations and complications including seroma, hematomas, abdominal wall abscess, wound infections, and mesh infections.

Results: Our sample population ( $\mathrm{n}=420$; mean age $46.3 \pm 11.7$ years) included 230 females (54.8\%), and cases included laparoscopic ( $\mathrm{n}=31 ; 7.5 \%)$, laparoscopic converted to open $(\mathrm{n}=7 ; 1.7 \%)$, and open $(\mathrm{n}=373,90 \%)$. As compared to suture repairs, mesh repair was associated with lower rates of complications ( $25.7 \%$ vs $29.5 \%$, $\mathrm{p}=0.10)$ and recurrence $(12.8 \%$ vs $15.2 \%, \mathrm{p}=0.67)$. Laparoscopic repairs had lower rates of complications than open repairs ( $25 \%$ vs $26.8 \%$; $\mathrm{p}=0.70$ ) but similar rates of recurrence $(13.8 \%$ and $13.6 \%$; $\mathrm{p}=0.53$ ). After logistic regression, obesity, chronic obstructive pulmonary disease, component separation technique, and prolonged operating time (>75th percentile) were associated with increased complications.
\end{abstract}

Conclusion: Obesity is a modifiable risk factor and must be addressed in patients undergoing VHRs. Mesh repair does not increase the risk of adverse long-term outcomes and may be performed safely in patients undergoing VHR.

Categories: Plastic Surgery, General Surgery

Keywords: abdomen ventral hernia, ventral wall hernias, hernia mesh

\section{Introduction}

Received 07/22/2020

Review began 07/24/2020

Review ended 07/25/2020

Published 08/02/2020

() Copyright 2020

Kadakia et al. This is an open access article distributed under the terms of the Creative Commons Attribution License CC-BY 4.0., which permits unrestricted use, distribution, and reproduction in any medium, provided the original author and source are credited.

Ventral hernia repair (VHR) is one of the most common general surgery procedures in the United States. More than 400,000 VHRs are performed annually [1]. These surgeries can be performed through open or laparoscopic techniques and with or without mesh.

Compared to the laparoscopic approach of VHR, open surgery has been associated with similar outcomes in mortality but mildly increased morbidity [2,3]. Additionally, mesh use has been associated with a lower risk for recurrence and a higher risk of infections [4,5]. Thus, mesh use is increasingly common in VHRs. Various factors such as mesh position and mesh placement technique may affect these complication rates. Intraperitoneal is the preferred mesh position in laparoscopic repairs; however, retrorectus placement of mesh may result in a lower recurrence rate and infections [6,7]. Certain comorbidities such as obesity, smoking history, and diabetes mellitus have also been associated with increased complications $[8,9]$. Preexisting hypertension is associated with increased rates of complications in abdominal surgical procedures overall; however, there is a lack of evidence on hypertension as a risk factor in hernia repairs, specifically [10].

Despite the abundant literature on outcomes of VHRs, few studies have a long-term follow-up. As a result, the long-term outcomes of VHRs, specifically recurrence and complications, are not well described. There is a need to identify major predictors of long-term complications, recurrences, and reoperations in patients undergoing VHRs to guide clinical practice. Such evidence may be used to guide specific practice approaches such as body mass index (BMI) cutoffs for surgery or the optimal duration for preoperative smoking cessation. These may also be useful to general surgeons in selecting the best method for surgical repair of ventral hernias. We now report factors associated with long-term outcomes, including recurrence, complications, and reoperation in patients undergoing VHRs.

\section{Materials And Methods}




\section{Patient selection}

After institutional review board (IRB) approval, a retrospective review of paper charts and electronic medical records of all patients over age 18 years, who underwent ventral incisional hernia repair at a single institution between January 1, 2000 and December 31, 2009, was performed. Only patients undergoing repair of primary hernias or incisional hernias with no more than one recurrence were included. Suprapubic and infraumbilical hernia repairs were excluded.

\section{Data collection}

Perioperative data collected included age, sex, BMI, type of hernia, size of defect, mesh position, mesh fixation technique, comorbidities (including hypertension, chronic obstructive pulmonary disease [COPD], previous abdominal surgery), and tobacco use. Postoperative data collected included postoperative urinary retention, length of hospital stay, and operative time. Prolonged operating time was defined as operating time greater than 75 th percentile (155 minutes).

\section{Independent variables}

Information about the size and type of repair was retrospectively collected. Laparoscopic repairs that were converted to open were classified as open repairs. The hernia defect size was defined as the largest width of the fascial as measured by the surgeon during the operation and was classified according to the European Hernia Society (EHS) classification system (W1 $<4 \mathrm{~cm}, \mathrm{~W} 2=4-10 \mathrm{~cm}$, W3 $\geqslant 10 \mathrm{~cm}$ ). For mesh repairs, mesh position was intraperitoneal, preperitoneal or other (onlay/sublay/inlay). Mesh fixation techniques included tacking, suturing, or other (glue, clips, staples, or unspecified technique). In cases with tacked and sutured fixation, the technique was classified as tacking. The use of component separation technique during repair was also noted.

\section{Outcomes}

The primary outcome was recurrence after VHR. Recurrence was defined as the occurrence of a ventral hernia in the same scar as previous VHR noted by a physician. Recurrence was primarily determined by either physical examination or CT scans. The secondary outcomes were reoperation and postoperative surgical complications. The complications included were grades I-IV according to the Clavien-Dindo classification system. Complications including seroma, hematoma, wound infection, and abdominal wall abscess were defined as those occurring after the repair and associated with the surgical site. Only complications that were likely to be related to the previous VHR based on the medical records and operative notes were included. Reoperation was defined as any operation after the repair for complications such as seroma, hematoma, wound infection, mesh infection, or abdominal wall abscess.

\section{Statistical analysis}

Statistical analysis was performed using SPSS version 24 (IBM Corp, Armonk, NY). To analyze the independent risk factors after the VHR repairs, we performed bivariable analyses screening clinically relevant risk factors, including age, sex, use of mesh, mesh fixation technique, mesh position, component separation, hernia size, and preoperation comorbidities such as diabetes, hypertension, tobacco use, COPD, and obesity. We used a t-test for continuous variables, Pearson's chi-square test for categorical variables, and Fisher's exact test for variables with an expected frequency less than 5. Univariate covariates were then entered simultaneously into a Cox regression model and multiple logistic regression model. Cox regression was used to evaluate the relationship between recurrence and clinical variables. The relationship between complications or reoperations and clinical variables was assessed using multivariate conditional regression analysis. All of the tests were two-tailed. $\mathrm{P}<0.05$ was considered to be significant.

\section{Results}

Between 2000 and 2009, 775 primary and incisional VHRs were performed at our institution. After excluding the repairs performed on patients with more than one VHR recurrence (129 cases) and infraumbilical/suprapubic repairs (148 cases), 420 patients were identified. During the follow-up period, 78 (18.57\%) patients were lost to follow-up. One patient died on postoperative day 13 due to peritonitis leading to septic shock. Four patients had postoperative urinary retention. We analyzed 420 patients (median age 46 years [range 38-54]) including 230 females (54.8\%). The procedures included were laparoscopic $(\mathrm{n}=31 ; 7.5 \%)$, laparoscopic converted to open $(n=7 ; 1.7 \%)$, and open $(n=372,90 \%)$. Mesh was used in $74.2 \%$ of all repairs. The median patient follow-up was 137 months.

\section{Characteristics of patients and repair}

Patient demographics and repair characteristics are shown in Table 1. Prevalence of comorbidities like hypertension, diabetes, COPD, tobacco use, and obesity were similar in all four groups. Mesh use was more common in larger hernia repairs $(p<0.0001)$ and was associated with longer operating times $(p=0.10)$. Laparoscopic technique was the preferred technique in larger hernia repairs $(\mathrm{p}<0.0001)$ although it was also with longer operating times $(\mathrm{p}=0.01)$. Mesh position was intraperitoneal in most open repairs ( $47.5 \% \mathrm{vs}$ $36.7 \%$ preperitoneal repairs). Mesh was tacked in most laparoscopic repairs but sutured in most open repairs. 


\section{Cureus}

The length of postoperative hospital stay was similar in all groups.

\begin{tabular}{|c|c|c|c|c|c|c|c|}
\hline Characteristic & Total $(\mathrm{N}=420)$ & Lap $(\mathrm{N}=31)$ & Open $(\mathrm{N}=389)$ & P Value & Mesh $(\mathrm{N}=312)$ & Suture $(\mathrm{N}=108)$ & P Value \\
\hline \multicolumn{8}{|l|}{ Sex } \\
\hline Female, n (\%) & $230(54.8)$ & $11(35.5)$ & $214(56.3)$ & \multirow{2}{*}{0.04} & $166(53.7)$ & $63(58.3)$ & \multirow{2}{*}{0.5} \\
\hline Male, n (\%) & $190(45.2)$ & $20(64.5)$ & $166(43.7)$ & & $143(46.3)$ & 45 (41.7) & \\
\hline Age, median (IQR) years & $46(38-54)$ & $46(33-51)$ & $46(38-54)$ & 0.89 & $46(38-55)$ & $47(39-53)$ & 0.63 \\
\hline BMI, median (IQR) & $\begin{array}{l}30.9(26.6- \\
36.5)\end{array}$ & $\begin{array}{l}31.7(27.3- \\
38.5)\end{array}$ & $30.8(26.5-36.3)$ & 0.97 & $30.8(27.0-36.5)$ & $31.2(25.8-36.6)$ & 0.86 \\
\hline Defect size, median (IQR) cm & $6(3-10)$ & $10(7-15)$ & $6(3-10)$ & $<0.0001$ & $8(4-10)$ & $4(1-8)$ & $<0.0001$ \\
\hline Tobacco use, n (\%) & $159(38.0)$ & 7 (22.6) & $152(39.7)$ & 0.08 & 118 (38.2) & 41 (37.6) & 0.91 \\
\hline Hypertension, n (\%) & $148(35.7)$ & $12(38.7)$ & $136(35.8)$ & 0.85 & $112(36.6)$ & $36(33.0)$ & 0.56 \\
\hline Diabetes, n (\%) & $72(17.6)$ & 4 (12.9) & $68(17.9)$ & 0.63 & $59(19.1)$ & $15(13.8)$ & 0.24 \\
\hline COPD, n (\%) & $41(9.8)$ & $2(6.5)$ & 39 (10.3) & 0.76 & $32(10.4)$ & $8(7.3)$ & 0.45 \\
\hline Previous abdominal surgery & $371(86.0)$ & 30 (96.8) & 335 (88.2) & 0.23 & 285 (92.2) & $86(78.9)$ & $<0.0001$ \\
\hline Follow-up, median (IQR) months & $137(111-168)$ & 157 (106-165) & $136(111-169)$ & 0.56 & $137(112-166)$ & $134(110-174)$ & 0.89 \\
\hline $\begin{array}{l}\text { Operating time, median (IQR) } \\
\text { minutes }\end{array}$ & $95(50-155)$ & 140 (95-205) & $90(50-100)$ & 0.01 & $102(60-160)$ & $72(35-130)$ & 0.1 \\
\hline Postop stay, median (IQR) days & $3(1-5)$ & $4(1-5)$ & $3(1-5)$ & 0.16 & $3(1-5)$ & $3(0-6)$ & 0.48 \\
\hline \multicolumn{8}{|l|}{ Mesh position, n (\%) } \\
\hline Intraperitoneal & $158(55.5)$ & $31(100)$ & $132(47.5)$ & \multirow{3}{*}{ - } & - & - & \multirow{3}{*}{-} \\
\hline Preperitoneal & $105(37.4)$ & NR & $101(36.7)$ & & - & - & \\
\hline Other* & $20(7.1)$ & NR & $44(15.8)$ & & - & - & \\
\hline \multicolumn{8}{|l|}{ Mesh fixation technique, $\mathrm{n}(\%)$} \\
\hline Tacked & $91(29.2)$ & $14(45.2)$ & 75 (27.5) & \multirow{2}{*}{ 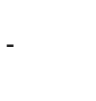 } & - & - & \\
\hline Sutured & $160(51.3)$ & $6(19.4)$ & $153(56)$ & & - & - & \\
\hline Unspecified/other ${ }^{+}$ & $61(19.6)$ & $11(35.5)$ & 53 (16.5) & & - & - & \\
\hline
\end{tabular}

\section{TABLE 1: Patient demographics}

*Onlay, sublay, inlay, or plug

+Other includes glue, clips, stymie pins, or not specified

BMI, body mass index; COPD, chronic obstructive pulmonary disease; IQR, interquartile range; Lap, laparoscopic

\section{Complications and reoperations}

Among complications, wound infections were most common (16.2\%), followed by seromas (11.3\%) (Table 2). Mesh infection was a complication in $4.9 \%$ of all mesh repairs $(n=312)$. The laparoscopic technique was associated with lower complications and reoperations rates as compared to the open technique. The overall complication rates for laparoscopic and open repairs were $25 \%$ and $26.8 \%$, respectively, whereas the reoperation rates were $6.9 \%$ and $13.2 \%$, respectively. However, upon multivariate analysis, no statistical difference was found in complication or reoperation rates between laparoscopic and open repairs ( $\mathrm{p}=0.70$ for complications and $\mathrm{p}=0.34$ for reoperations). 


\section{Cureus}

\begin{tabular}{|l|l|l|l|l|l|l|l|}
\hline Outcomes (\%) & Total $(\mathbf{N}=\mathbf{4 2 0})$ & Lap $(\mathbf{N}=\mathbf{3 1})$ & Open $(\mathbf{N}=\mathbf{3 8 9})$ & P Value & Mesh $(\mathbf{N}=\mathbf{3 1 2})$ & Suture (N=108) & P Value \\
\hline Any complication & $26.6 \%$ & $25.0 \%$ & $26.8 \%$ & $0.70^{\star}$ & $25.7 \%$ & $29.5 \%$ & $0.10^{\star}$ \\
\hline Wound infection (including mesh infection) & $16.8 \%$ & $7.1 \%$ & $17.6 \%$ & 0.19 & $14.9 \%$ & $22.5 \%$ & 0.09 \\
Seroma & $11.3 \%$ & $21.4 \%$ & $10.5 \%$ & 0.17 & $12.0 \%$ & $9.5 \%$ & 0.24 \\
Hematoma & $3.9 \%$ & $0.0 \%$ & $4.3 \%$ & 0.40 & $4.3 \%$ & $2.9 \%$ & 0.48 \\
Abdominal wall abscess & $10.3 \%$ & $3.6 \%$ & $11.1 \%$ & 0.34 & $9.5 \%$ & $12.7 \%$ & 0.35 \\
Reoperation & $12.8 \%$ & $6.9 \%$ & $13.2 \%$ & $0.34^{\star}$ & $13.1 \%$ & $10.8 \%$ & $0.75^{\star}$ \\
Recurrence & $13.6 \%$ & $13.8 \%$ & $13.6 \%$ & $0.53^{\star}$ & $12.8 \%$ & $15.2 \%$ & $0.67^{\star}$ \\
\hline
\end{tabular}

\section{TABLE 2: Ventral hernia repair outcomes}

*Adjusted p-values after logistic regression

Lap, laparoscopic

Logistic regression analysis for complications and reoperations is summarized in Table 3. VHRs with mesh had lower rates of complications ( $25.7 \%$ vs $29.5 \%$ for suture repairs) and slightly higher rates of reoperation ( $13.1 \%$ vs $10.8 \%$ for primary repairs). However, these differences were not significant after multivariate adjustment with logistic regression. After multivariate analysis, obesity, COPD, component separation technique, and prolonged operating time ( $>75$ th percentile) were associated with an increased risk of complications. Patients with BMI $\geqslant 30$ had an odds ratio (OR) of 2.13 (95\% CI 1.24 to 3.67 ; $\mathrm{p}=0.01$ ) for developing complications like wound infection, seroma, hematoma, and abdominal wall abscess. Patients with COPD had an OR of 2.29 (95\% CI 1.06 to 4.96; p=0.04) for developing complications. There were no significant interactions for analysis of complication rates. Use of component separation technique was associated with increased risk of complications (OR 5.67, 95\% CI 1.04 to 31.08; $\mathrm{p}=0.05$ ) and reoperations (OR $15.64,95 \%$ CI 2.86 to 85.86 ; $\mathrm{p}<0.001$ ). 


\section{Cureus}

\begin{tabular}{|c|c|c|c|c|}
\hline \multirow{2}{*}{ Variables } & \multicolumn{2}{|l|}{ Complication } & \multicolumn{2}{|l|}{ Reoperation } \\
\hline & OR (95\% Cl) & P Value & OR $(95 \% \mathrm{Cl})$ & P Value \\
\hline Sex (male) & $1.51(0.85-2.68)$ & 0.16 & $1.61(0.75-3.49)$ & 0.22 \\
\hline Age (one year older) & $1.00(0.98-1.02)$ & 0.98 & $1.00(0.97-1.03)$ & 0.87 \\
\hline $\mathrm{BMI} \geq 30$ & $2.13(1.24-3.67)$ & 0.01 & $1.48(0.71-3.07)$ & 0.30 \\
\hline Tobacco use & $0.86(0.51-1.45)$ & 0.57 & $0.78(0.38-1.58)$ & 0.49 \\
\hline Hypertension & $1.49(0.87-2.54)$ & 0.15 & $0.90(0.42-1.95)$ & 0.79 \\
\hline COPD & $2.29(1.06-4.96)$ & 0.04 & $2.10(0.78-5.64)$ & 0.14 \\
\hline Previous abdominal surgery & $2.31(0.92-5.80)$ & 0.08 & $0.91(0.31-2.72)$ & 0.87 \\
\hline Wound class (dirty) & $0.86(0.44-1.67)$ & 0.66 & $1.07(0.45-2.57)$ & 0.88 \\
\hline Mesh use & $0.62(0.35-1.11)$ & 0.10 & $1.13(0.50-2.53)$ & 0.77 \\
\hline Lap vs open & $0.83(0.32-2.18)$ & 0.70 & $0.49(0.10-2.28)$ & 0.36 \\
\hline Component separation & $5.67(1.04-31.08)$ & 0.05 & $15.27(2.78-83.74)$ & $<0.0001$ \\
\hline Prolonged operating time & $3.01(1.71-5.31)$ & $<0.0001$ & $2.22(1.04-4.75)$ & 0.04 \\
\hline Defect size $>4 \mathrm{~cm}$ & $0.57(0.3-1.07)$ & 0.08 & $0.61(0.27-1.41)$ & 0.25 \\
\hline
\end{tabular}

TABLE 3: Multivariate analyses of risk factors for complications and reoperations after ventral hernia repair using logistic regression

BMI, body mass index; COPD, chronic obstructive pulmonary disease; Lap, laparoscopic

\section{Recurrence rates}

During the follow-up period, $13.6 \%$ of patients overall had at least one recurrence (Table 2). VHRs with mesh had lower rates of recurrence as compared to suture repairs ( $12.8 \%$ vs $15.2 \%$, respectively; $\mathrm{p}=0.67$ ); however, this difference was not statistically significant after Cox regression analysis. There was no significant difference in patients undergoing repair with laparoscopic or open technique (13.6\% and $13.8 \%$, respectively). The best-fitting Cox regression model included sex, age, obesity, tobacco use, hypertension, COPD, previous abdominal surgery, operative technique (laparoscopic vs open), mesh use, component separation technique, and prolonged operating time (Table 4). No significant risk factors for recurrence were identified after Cox regression analysis. 


\section{Cureus}

\begin{tabular}{|c|c|c|}
\hline \multirow{2}{*}{ Variables } & \multicolumn{2}{|l|}{ Recurrence } \\
\hline & HR (95\% Cl) & P Value \\
\hline Sex (male) & $0.73(0.390-1.34)$ & 0.31 \\
\hline Age (one year older) & $0.99(0.97-1.02)$ & 0.83 \\
\hline $\mathrm{BMI} \geq 30$ & $1.66(0.92-2.99)$ & 0.09 \\
\hline Tobacco use & $1.36(0.77-2.38)$ & 0.29 \\
\hline Hypertension & $1.03(0.57-1.84)$ & 0.93 \\
\hline COPD & $1.65(0.78-3.51)$ & 0.19 \\
\hline Previous abdominal surgery & $1.39(0.54-3.72)$ & 0.50 \\
\hline Mesh use & $0.74(0.40-1.35)$ & 0.32 \\
\hline Lap vs open & $1.12(0.41-3.40)$ & 0.75 \\
\hline ponent separation & $2.17(0.51-9.82)$ & 0.30 \\
\hline
\end{tabular}

\section{TABLE 4: Cox regression analysis of risk factors for recurrence}

BMI, body mass index; COPD, chronic obstructive pulmonary disease; Lap, laparoscopic

\section{Discussion}

In our retrospective study of 420 patients who underwent primary or incisional VHRs between 2000 and 2009, we found that VHRs with laparoscopic technique and mesh use had lower rates of complications; however, this difference was not statistically significant after multivariate analysis. After a median followup time of 137 months, patients who underwent repair with mesh had a lower rate of recurrence $(\mathrm{p}=0.67)$. Furthermore, we identified obesity, COPD, prolonged operating time, and component separation technique as significant risk factors for complications, such as seroma, hematoma, wound infections, and abdominal wall abscess.

Our results, indicating the lack of differences in outcomes of laparoscopic and open repairs, are consistent with prior studies with similar long follow-up times, demonstrating that recurrence and complication rates did not differ between open and laparoscopic techniques [11-13]. A meta-analysis of randomized controlled trials showed that recurrence rates were similar for both techniques [13]. Another prospective study with long-term comparison of 710 repairs showed that quality of life (in short term), length of stay, and infection rates were decreased in laparoscopic repairs; however, overall long-term complications and recurrence rates were equal [14]. Previous studies have reported that mesh use, compared to suture repair, is associated with a lower risk of hernia recurrence and increased risk of infection [6]. Our study found lower rates of long-term recurrence and complications in patients who underwent VHR with mesh. Although these differences were not statistically significant, the differences in recurrence rates in our study are similar to those reported in literature. The lack of significance in our study can be attributed to low sample size.

Additionally, our analysis indicates higher rates of complications and reoperations in VHR with use of component separation technique. These results are likely attributable to the increased wound-healing complications and the already large defect size. A study of patients undergoing large VHRs showed that component separation technique may be ideal hernia repair for large defects; however, our results indicate significantly higher adverse outcomes of component separation technique in open VHRs $[15,16]$.

Our findings are consistent with previous studies showing that obesity increases risk of complications after VHR [17,18]. A retrospective chart review study of 888 patients was analyzed to develop a risk-stratification scoring system for surgical site complications after VHR [18]. This study reported that a BMI $\geqslant 40$ had an OR of 3.2 for the development of surgical site infection, and mesh implant had an OR of 1.9 for the development of infection, seroma, hematoma, wound dehiscence, and/or fistula formation. Thus, there is a consensus on higher complication rates after VHR in morbidly obese patients, $\mathrm{BMI} \geqslant 40$; however, our analysis indicates that obese patients (BMI $\geqslant 30$ ) undergoing VHR have an OR of 2.13 (95\% CI 1.24 to 3.67, p=0.01) of postoperative complications $[8,18]$. The mechanisms underlying the increased complication rate in obese patients are likely multifactorial, but hypotheses exist that may explain the predisposition for complications in obese and hypertensive patients [10]. Impaired visualization due to body habitus in addition to defects in tissue structure and healing are likely contributors to postoperative complications after VHRs in obese patients [19]. 
Limitations of this study include small sample size, treatment facility bias, retrospective review, and significant loss of information due to data extraction from paper charts. Other data, such as the presence of multiple defects, location of hernia (for example, midline vs lateral), suture type, mesh types, preoperative medications, number of surgeons operating, and emergency vs elective procedures, were not available to be included in this study. Additionally, all patients had variable follow-up periods. In our Cox regression model, essential covariates like obesity lost significance because of our small sample size. Furthermore, mortality among the patients who were lost to follow-up was unknown. Therefore, it was not possible to report the overall mortality in this study.

Taken together, these findings suggest that variability in surgical outcomes exists for VHR in patients with obesity and preexisting hypertension. Addressing these modifiable risk factors in surgical candidates preoperatively may reduce the risk of complications and may guide the decision to undergo and timing of surgery.

\section{Conclusions}

For patients undergoing VHR, obesity and COPD are significant risk factors for complications. Laparoscopic repairs had slightly lower rates of complication but similar rates of recurrence as open repairs after a longterm follow-up. Mesh repairs had lower rates of overall complications and recurrence. Mesh repairs should be considered in all patients, given that their complication rates were lower in our study, although not statistically significant. This study was limited by the number of patients with a complete follow-up available for analysis. Future studies looking into recurrence and complication rates in a larger, matched cohort may help confirm if long-term outcomes reported in this study are similar to those in the population. Obesity, COPD, and use of component separation technique are independent risk factors for complications after VHRs. Obesity is a modifiable risk factor, and future studies focused on these risk factors are necessary to revise treatment guidelines for VHR candidates with these comorbidities.

\section{Additional Information}

\section{Disclosures}

Human subjects: All authors have confirmed that this study did not involve human participants or tissue. Animal subjects: All authors have confirmed that this study did not involve animal subjects or tissue. Conflicts of interest: In compliance with the ICMJE uniform disclosure form, all authors declare the following: Payment/services info: All authors have declared that no financial support was received from any organization for the submitted work. Financial relationships: All authors have declared that they have no financial relationships at present or within the previous three years with any organizations that might have an interest in the submitted work. Other relationships: All authors have declared that there are no other relationships or activities that could appear to have influenced the submitted work.

\section{References}

1. Huerta S, Varshney A, Patel PM, Mayo HG, Livingston EH: Biological mesh implants for abdominal hernia repair: US Food and Drug Administration approval process and systematic review of its efficacy. JAMA Surg. 2016, 151:374-381. 10.1001/jamasurg.2015.5234

2. Zhang Y, Zhou H, Chai Y, Cao C, Jin K, Hu Z: Laparoscopic versus open incisional and ventral hernia repair: a systematic review and meta-analysis. World J Surg. 2014, 38:2233-2240. 10.1007/s00268-014-2578-Z

3. Aher C V, Kubasiak JC, Daly SC, et al.: The utilization of laparoscopy in ventral hernia repair: an update of outcomes analysis using ACS-NSQIP data. Surg Endosc. 2015, 29:1099-1104. 10.1007/s00464-014-3798-X

4. Christoffersen MW, Helgstrand F, Rosenberg J, Kehlet H, Bisgaard T: Lower reoperation rate for recurrence after mesh versus sutured elective repair in small umbilical and epigastric hernias. A nationwide register study. World J Surg. 2013, 37:2548-2552. 10.1007/s00268-013-2160-0

5. Kokotovic D, Bisgaard T, Helgstrand F: Long-term recurrence and complications associated with elective incisional hernia repair. JAMA. 2016, 316:1575-1582. 10.1001/jama.2016.15217

6. Holihan JL, Hannon C, Goodenough C, et al.: Ventral hernia repair: a meta-analysis of randomized controlled trials. Surg Infect. 2017, 18:647-658. 10.1089/sur.2017.029

7. Albino FP, Patel KM, Nahabedian MY, Sosin M, Attinger CE, Bhanot P: Does mesh location matter in abdominal wall reconstruction? A systematic review of the literature and a summary of recommendations. Plast Reconstr Surg. 2013, 132:1295-1304. 10.1097/PRS.0b013e3182a4c393

8. Liang MK, Holihan JL, Itani K, et al.: Ventral hernia management: expert consensus guided by systematic review. Ann Surg. 2017, 265:80-89. 10.1097/SLA.0000000000001701

9. Kaoutzanis C, Leichtle SW, Mouawad NJ, Welch KB, Lampman RM, Wahl WL, Cleary RK : Risk factors for postoperative wound infections and prolonged hospitalization after ventral/incisional hernia repair. Hernia. 2015, 19:113-123. 10.1007/s10029-013-1155-y

10. Koolen PGL, Ibrahim AMS, Kim K, et al.: Patient selection optimization following combined abdominal procedures: analysis of 4925 patients undergoing panniculectomy/abdominoplasty with or without concurrent hernia repair. Plast Reconstr Surg. 2014, 134:539-550. 10.1097/PRS.0000000000000519

11. Ballem N, Parikh R, Berber E, Siperstein A: Laparoscopic versus open ventral hernia repairs: 5 year recurrence rates. Surg Endosc. 2008, 22:1935-1940. 10.1007/s00464-008-9981-1

12. Bingener J, Buck L, Richards M, Michalek J, Schwesinger W, Sirinek K: Long-term outcomes in laparoscopic vs open ventral hernia repair. Arch Surg. 2007, 142:562-567. 10.1001/archsurg.142.6.562

13. Sajid MS, Bokhari SA, Mallick AS, Cheek E, Baig MK: Laparoscopic versus open repair of incisional/ventral 


\section{Cureus}

hernia: a meta-analysis. Am J Surg. 2009, 197:64-72. 10.1016/j.amjsurg.2007.12.051

14. Colavita PD, Tsirline VB, Belyansky I, Walters AL, Lincourt AE, Sing RF, Heniford BT: Prospective, long-term comparison of quality of life in laparoscopic versus open ventral hernia repair. Ann Surg. 2012, 256:714-723. 10.1097/sla.0b013e3182734130

15. Dumanian GA, Denham W: Comparison of repair techniques for major incisional hernias . Am J Surg. 2003, 185:61-65. 10.1016/S0002-9610(02)01122-4

16. Ko JH, Wang EC, Salvay DM, Paul BC, Dumanian GA: Abdominal wall reconstruction: lessons learned from 200 “components separation” procedures. Arch Surg. 2009, 144:1047-1055. 10.1001/archsurg.2009.192

17. Shankar DA, Itani KMF, O’Brien WJ, Sanchez VM: Factors associated with long-term outcomes of umbilical hernia repair. JAMA Surg. 2017, 152:461-466. 10.1001/jamasurg.2016.5052

18. Berger RL, Li LT, Hicks SC, Davila JA, Kao LS, Liang MK: Development and validation of a risk-stratification score for surgical site occurrence and surgical site infection after open ventral hernia repair. J Am Coll Surg. 2013, 217:974-982. 10.1016/j.jamcollsurg.2013.08.003

19. Hedenstierna G, Larsson A: Influence of abdominal pressure on respiratory and abdominal organ function Curr Opin Crit Care. 2012, 18:80-85. 10.1097/MCC.0b013e32834e7c3a 